\title{
The Optimal Anti-Thrombotic Regimen after Surgical Bioprosthetic Aortic Valve Replacement-Does it Really Matter?
}

\author{
Constantin von zur Mühlen ${ }^{1}$ Wolfgang Bothe ${ }^{2}$ Christoph Bode ${ }^{1}$
}

${ }^{1}$ Department of Cardiology and Angiology I, University Heart Center Freiburg, Faculty of Medicine, University of Freiburg, Freiburg, Germany

2 Department of Cardiovascular Surgery, University Heart Center Freiburg, Medical Faculty, University of Freiburg, Freiburg, Germany

Thromb Haemost 2019;119:189-190.

Leaflet thrombosis was an unexpected finding after transcatheter aortic valve implantation (TAVI). The clinical significance of this phenomenon is not yet completely clear; however, it has raised questions about the optimal periprocedural and long-term anti-thrombotic strategy.

So what can we learn from surgical bioprosthetic aortic valve replacement (BAVR)? An et al are to be congratulated for shedding some light into this black box with the article published in this issue of the journal. ${ }^{1}$ Surprisingly, the question which anti-thrombotic regimen after BAVR is best for the patient, an anti-platelet-based therapy or an anticoagulation-based therapy, is still unresolved after decades of BAVR. Balancing the risks of bleeding and thromboembolic events is getting more important for BAVR patients, since especially patients at younger age and with low operative risk are candidates for surgical aortic valve replacement (SAVR). Most recent guidelines of the European Society of Cardiology give recommendations about the treatment strategy with regard to the selection of SAVR or TAVI. ${ }^{2}$ Besides anatomical and technical considerations, age and Society of Thoracic Surgery (STS) risk score are key factors helping the interdisciplinary heart team to decide whether SAVR or TAVI are more suitable for the patients with aortic valve stenosis. For now, in patients with low STS risk score and younger age, SAVR is still the therapy of choice. After successful SAVR, these patients have an average remaining live expectancy of more than one decade-thus, safe and effective long-term anti-thrombotic therapy is of utmost importance.

The systematic review by An et al summarizes all available data that address this crucial question. The authors performed a rigorous systematic review, which could only be based on two randomized controlled trials (RCTs; $n=397$ patients) and five observational studies ( $n=2,012$ patients). The mean follow-up for all outcomes was only 3 months in

Address for correspondence Constantin von zur Mühlen, MD, Department of Cardiology and Angiology I, University Heart Center Freiburg-Bad Krozingen, Hugstetter Strasse 55, 79106 Freiburg, Germany

(e-mail: constantin.vonzurmuehlen@universitaets-herzzentrum.de).

RCTs, and 10 months for observational studies. Anti-platelet compared with anticoagulant therapy demonstrated a trend toward fewer major bleedings in RCTs $(p=0.06)$, and significantly fewer major bleedings in observational studies $(p<0.0001)$, while stroke, thromboembolism and mortality did not show a significant difference in either RCTs or observational studies. As a conclusion from this review, anti-platelet therapy demonstrated reduced bleeding risk with no negative effects on stroke, thromboembolism or mortality compared with anticoagulation therapy after surgical BAVR. As the authors emphasize, their confidence in the results was reduced by the low quality of the available evidence.

We recently described the situation of SAVR in Germany in the context of increasing numbers of TAVI procedures. ${ }^{3,4}$ While the number of TAVI procedures continuously increased from 2009 to 2015, especially in patient groups of intermediate and high operative risk, the number of SAVR was only slightly decreasing (2009: 8,259 SAVR; 2015: 6,226 SAVR)-with the highest proportion of SAVR in younger patients below 75 years of age.

So, especially in the context of a largely missing and shaky database: how are patients actually treated? The authors report on an interesting international survey from 2008, conducted in 48 centres. Clinicians prescribed warfarin in $43 \%$ of centres, aspirin (ASA) in $33 \%$ of centres, the combination of warfarin and ASA in $20 \%$ of centres and no antithrombotic therapy in $4 \%$ of centres. ${ }^{5}$

What can we then learn from most recent clinical trials? First insights from the GALILEO study, although not yet published at the time this editorial was written, are now available. Patients after TAVI were either treated with a rivaroxaban-based strategy of $10 \mathrm{mg}$ rivaroxaban +75 to $100 \mathrm{mg}$ of ASA for 3 months, followed by ASA only, versus a

(c) 2019 Georg Thieme Verlag KG Stuttgart · New York
DOI https://doi.org/ 10.1055/s-0038-1677504. ISSN 0340-6245.
September 30, 2018

accepted after revision

December 17, 2018 


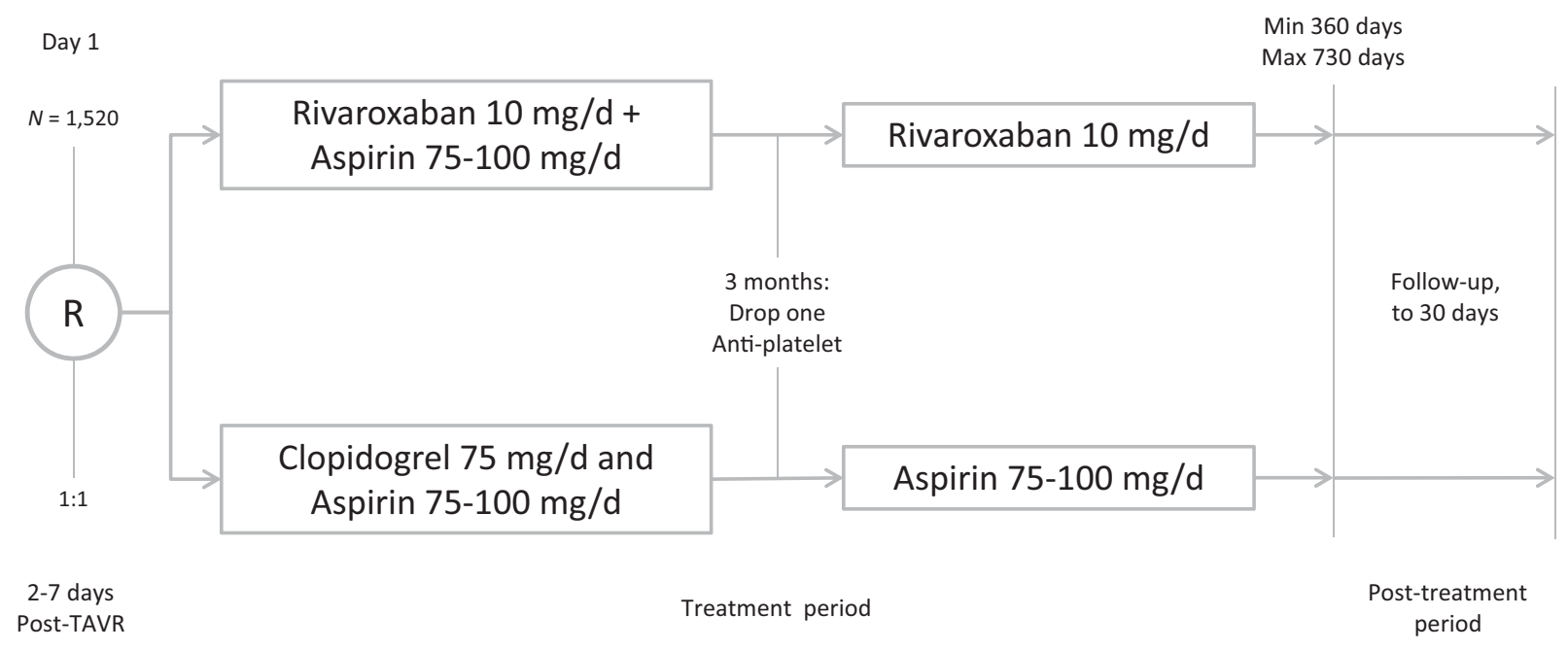

Fig. 1 Study design of the GALILEO study (Global Study Comparing a rivAroxaban-based Antithrombotic Strategy to an antipLatelet-based Strategy After Transcatheter aortlc vaLve rEplacement to Optimize Clinical Outcomes). Primary efficiency endpoints: composite of death, stroke, MI, symptomatic valve thrombosis, systemic thromboembolism, or major VTE. ${ }^{7}$

dual anti-platelet therapy (DAPT) with clopidogrel $75 \mathrm{mg} /$ d + ASA 75 to $100 \mathrm{mg}$ for 3 months, followed by ASA only (see - Fig. 1). ${ }^{6,7}$ Importantly, patients had no background of atrial fibrillation. The study was stopped prematurely in August 2018 by the Data Safety Monitoring Board, since the rate of death and time to first thromboembolic event differed between the groups (11.4\% in the rivaroxaban/ASAbased group vs. $8.8 \%$ in the DAPT group), and mortality was doubled in the rivaroxaban/ASA group compared with the DAPT group (6.8\% vs. $3.3 \%$ ). Many questions regarding the detailed outcomes of GALILEO remain unanswered at the moment. Also, these patients were treated non-surgically with a bioprosthesis-based TAVI system, and we do not know if these findings can be transferred into the world of BAVR. Quite obviously, we need more and much better data for an evidence-based approach in the future.

But what is the conclusion for now? We only have minor evidence from two RCTs available at the moment, showing a trend in favour of the anti-platelet therapy with respect to a reduced bleeding risk. Observational studies show that antiplatelet therapy reduces bleeding risk with no negative effects on stroke, thromboembolism or mortality compared with anticoagulation therapy after surgical BAVR. For all we can extrapolate from recent randomized trials with novel oral anticoagulants, we cannot expect a solution from this approach. Therefore, for now, an anti-platelet approach appears to be the most reasonable strategy.
Conflict of Interest

None declared.

\section{References}

1 An KR, Belley-Cote EP, Um KJ, et al. Anti-platelet therapy versus anticoagulation after surgical bioprosthetic aortic valve replacement: a systematic review and meta-analysis. Thromb Haemost 2019;119(02). Doi: 10.1055/s-0038-1676816

2 Baumgartner H, Falk V, Bax JJ, et al; ESC Scientific Document Group. 2017 ESC/EACTS Guidelines for the management of valvular heart disease. Eur Heart J 2017;38(36):2739-2791

3 Reinöhl J, Kaier K, Reinecke $\mathrm{H}$, et al. Effect of availability of transcatheter aortic-valve replacement on clinical practice. N Engl J Med 2015;373(25):2438-2447

4 Stachon P, Kaier K, Zirlik A, et al. Development and results of transcatheter and surgical aortic valve replacement in Germany 2014 and 2015 [in German]. Dtsch Med Wochenschr 2018;143 (23):e206-e212

5 Colli A, Verhoye JP, Heijmen R, et al; ACTION Registry Investigators. Antithrombotic therapy after bioprosthetic aortic valve replacement: ACTION Registry survey results. Eur J Cardiothorac Surg 2008;33(04):531-536

6 Hemmrich M, Peterson ED, Thomitzek K, Weitz JI. Spotlight on unmet needs in stroke prevention: the PIONEER AF-PCI, NAVIGATE ESUS and GALILEO trials. Thromb Haemost 2016;116(Suppl 2): S33-S40

7 Windecker S, Tijssen J, Giustino G, et al. Trial design: rivaroxaban for the prevention of major cardiovascular events after transcatheter aortic valve replacement: rationale and design of the GALILEO study. Am Heart J 2017;184:81-87 